\title{
A simplified approach to the management of tangential retinal traction bands
}

\author{
GHOLAM A. PEYMAN, JAMES L. GREEN, AND C. PATRICK CARROLL \\ From the Department of Ophthalmology, University of Illinois Hospital Eye and Ear Infirmary, \\ Chicago, USA
}

SUMMARY We describe a simple technique for cutting preretinal fibrovascular traction bands. The instrumentation includes a vitrophage and a membrane dissector connected to a bipolar diathermy unit. The traction bands are rubbed between the membrane dissector and the vitrophage while they are simultaneously coagulated until they are transsected.

Closed pars plana surgery was confined initially to the removal of opaque vitreous gel after vitreous haemorrhage. By modifying the cutting port of the vitrectomy instrument, ${ }^{1}$ taut intravitreal bands and thick secondary membranes could be severed. Because of their proximity to the retina, preretinal membranes and tangential retinal traction bands were difficult to manage. Although the use of a hooked needle ${ }^{2}$ facilitated the lifting of these bands from the surface of the retina, it was difficult to sever them with automated vitrectomy instruments or scissors. Scissors had to be miniaturised and their blades angulated to reach the bands from the proper direction. $^{3-5}$ Often the surgeon had to insert one instrument inside the eye and exchange it later for another, a manoeuvre that increased the risk of inadvertent lens injury and retinal dialysis. This report describes a simplified technique for the management of tangential traction bands with use of bipolar diathermy in conjunction with a fibreoptic membrane dissector and a vitrectomy instrument.

\section{Materials and methods}

\section{INSTRUMENTS}

The method of releasing retinal traction was devised for use with the 2-instrument technique, bipolar wetfield cautery, and intraocular illumination. The vitrectomy instrumentation preferred by the surgeon and the dissector are connected to a bipolar diathermy unit with alligator clips.

We use a wide-angle cutter vitrophage (or miniphage) and a Peyman membrane dissector (Fig. 1). The dissector is combined with a fibreoptic light

Correspondence to Dr G. A. Peyman, University of Illinois Hospital Eye and Ear Infirmary, 1855 W Taylor St., Chicago, IL 60612, USA.

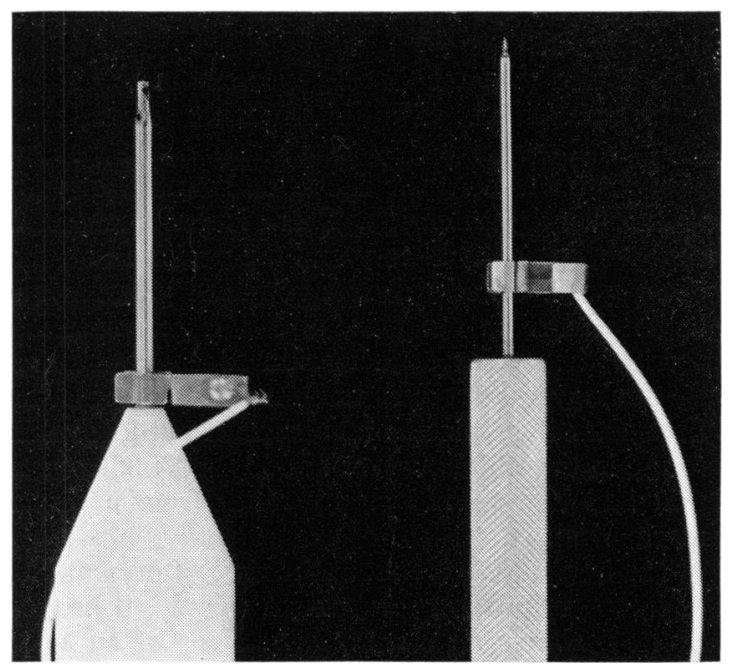

Fig. 1 Wide-angle cutter vitrophage (left). Combination fibreoptic illuminator and Peyman membrane dissector (right). Each instrument is attached to wet-field cautery by leads.

source for intraocular illumination. The dissector has a long sharp tip that is angled at approximately $100^{\circ}$ to the shaft of the intraocular illuminator.

\section{OPERATIVE PROCEDURE}

The eye is prepared as described previously for a pars plana vitrectomy. ${ }^{6}$ A second $2 \mathrm{~mm}$ long sclerotomy is then made approximately $160^{\circ}$ away from the primary sclerotomy site and $3.5 \mathrm{~mm}$ posterior to the limbus. The second sclerotomy incision is made to accommodate the fibreoptic light pick or the membrane dissector. A vitrectomy is then performed until all circumferential and anteroposterior traction 


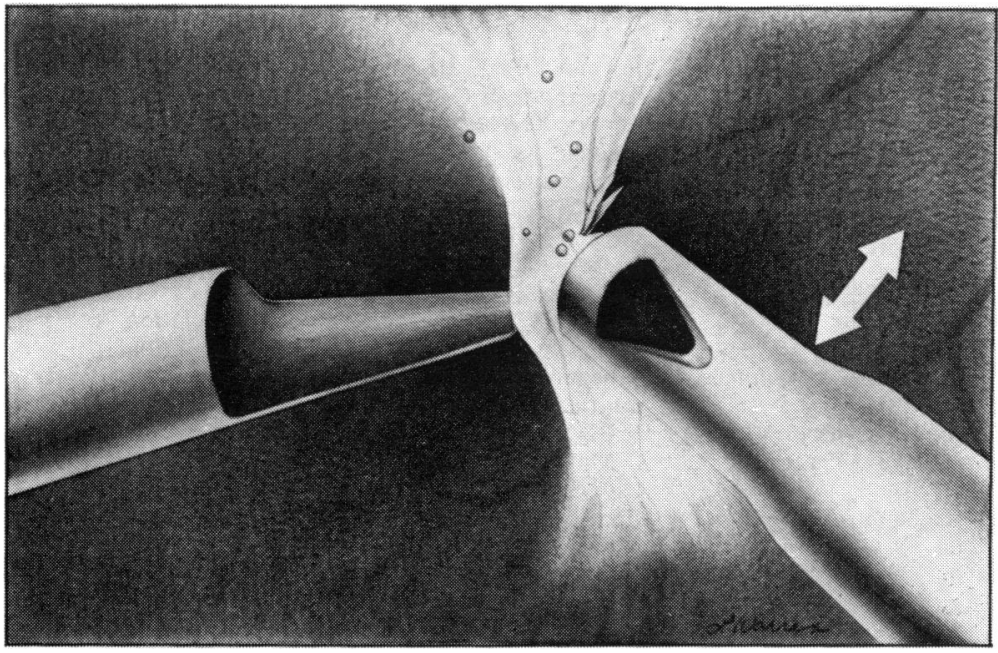

Fig. 2 Schematic presentation of membrane sectioning. Fibreoptic membrane dissector (left) is held stationary. Membrane is rubbed between wide-angle cutter vitrophage and the dissector while current is passed through the membrane.

is relieved. Taut fibrous bands are dissected until a free space is developed between the band and the retinal surface; this is done easily with the membrane dissector. The membrane dissector is then run along the length of the band, and light diathermy is applied to close vessels that may be contained within the band. As the fibreoptic membrane dissector is held stationary above the surface of the retina, the surgeon carefully rubs the vitrophage over the traction band, while current flow is gradually increased between the 2 instruments (Fig. 2). Traction bands, which previously could be cut only with vitreous scissors, are seen to melt away from the effect of rubbing and cauterisation. In addition any vessels that remain patent are closed.

Caution should be taken so that (1) the dissector remains stationary and slightly above the surface of the retina to prevent coagulation of the underlying retina and formation of tears; (2) coagulation should be started on a low setting and slowly advanced, for too high a setting can cause bubble formation that obscures the view and disrupts the tissue with possible haemorrhage; and (3) the fibrous bands must lie between the 2 instruments. If the instruments touch, current will pass through the instruments and bypass the tissue to be cut.

\section{Discussion}

Many different types of surgical instruments, picks, and needles have been designed to manipulate and cut vitreous opacities and traction bands. These instruments often have to be exchanged for scissors when cutting preretinal traction bands. Repeated exchange of instruments inside the eye enhances the chance of retinal dialysis at the site of the sclerotomy.
Mechanical scissors tend to push away relatively taut fibrous bands rather than cut them. When scissors are used in place of a vitrectomy instrument, visualisation can be compromised if any bleeding occurs, because there is no constant exchange of intraocular fluid.

Peyman, ${ }^{7}$ Shea, ${ }^{8}$ and Schepens et al. ${ }^{9}$ have developed under-water diathermy probes that are capable of coagulating intraocular vessels. While effective in stopping intraocular haemorrhage, these instruments have not been effective cutting tools.

O'Malley and Heintz ${ }^{10}$ developed an instrument to perform 'electrovitrectomy'. This method of performing vitrectomy and severing traction bands suffered from many difficulties, including the rapid loss of cutting efficiency owing to the accumulation of debris on the tip of the instrument and the inability to cut taut vitreous bands except with great difficulty. Charles and associates ${ }^{11}$ have also described a method of performing intraocular coagulation by connecting each of 2 intraocular instruments to a wet-field cautery. ${ }^{12}$ This proved to be an extremely convenient and effective way of handling intraocular bleeding. We are unaware of anyone using the wet-field cautery to cut membranes.

By combining the technique of membrane dissection with 2 intruments and bipolar intraocular wet-field cautery we have developed a technique to cut tractional bands. Vascularised membranes closely apposed to the retina are cut safely after being dissected away from the retinal surface. This technique is advantageous in that (1) it eliminates the need to interchange instruments during vitrectomy; (2) the bands can be approached with ease from various angles; and (3) the vascular portion of the membrane is coagulated as the band is being cut. 
The only disadvantage we have found is the occasional accumulation of coagulated material on the tip of the dissector. The material, however, is easily cleaned by the vitrophage without removing either instrument from the eye.

Supported in part by core grant 1P30EY01792 and training grant EY703802 from the National Institutes of Health, Bethesda, Md.

\section{References}

1 Peyman GA. Wide-angle cutter vitrophage. Ophthalmic Surg 1976; 7: 96-7.

2 Machemer R. A new concept for vitreous surgery: VII. Two-instrument technique in pars plana vitrectomy. Arch Ophthalmol 1974; 92: 407-12.

3 Neubauer H. Management of non-magnetic intraocular foreign bodies. In: Freeman HM, ed. Ocular Trauma. New York: Appleton-Century-Crofts, 1979: 187-96.

4 Meredith TA, Kaplan HJ, Aaberg TM. Pars plana vitrectomy: techniques for relief of epiretinal traction by membrane segmentation. Am $J$ Ophthalmol 1980; 83: 408-13.

5 Peyman GA, Huamonte FU. Vitreous scissors and forceps. Am J Ophthalmol 1975; 80: 767.

6 Peyman GA, Huamonte FU, Goldberg MF, Sanders DR, Nagpal KC, Raichand M. Four hundred pars plana vitrectomies with the vitrophage. Arch Ophthalmol 1978; 96: 45-50.

7 Peyman GA. Radio frequency infusion probe: an instrument for intraocular diathermy. Ophthalmic Surg 1972; 3: 32-7.

8 Shea MC. Intravitreal electrocoagulation of retinal vessels. Can J Ophthalmol 1972; 7: 268-70.

9 Schepens C, Delori F, Rogers F, Constable I. An optimized underwater diathermy. Ophthalmic Surg 1975; 6: 32-6.

10 O'Malley C, Heintz EA. Electrovitrectomy. Am J Ophthalmol 1973; 76: 336-41.

11 Charles S, White J, Dennison C, Eichenbaum D. Bimanual bipolar intraocular diathermy. Am J Ophthalmol 1976; 81: 101-3.

12 McPherson SD. Bipolar coagulation in ophthalmic operations. Am J Ophthalmol 1972; 73: 790-1. 